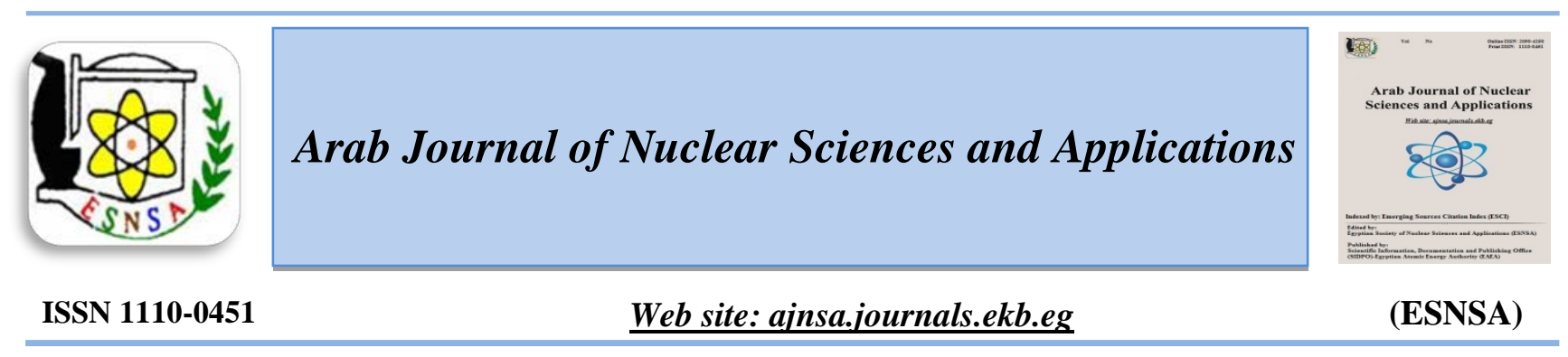

\title{
A Comparative Study of Volumetric Brain Tumor Measurements Using Different Manual Methods
}

\author{
Mohammed Elywa \\ Biophysics Branch, Physics Department, Faculty of Science, Zagazig University, 44519 Zagazig, Egypt \\ Corresponding author E-mail: elywa2006@yahoo.com
}

\begin{abstract}
Received $14^{\text {th }}$ Jun 2017 Early detection of a minor tumor size change is difficult using traditional visual and clinical Accepted $28^{\text {th }}$ Aug 2017 examination measurements. Although they are time-consuming, the general manual methods are the standard approach for quantitative measurement of human tumor and used in several hospitals. Magnetic Resonance Imaging (MRI) techniques are used to measure the human tumor volume manually or automatically. Three mathematical manual methods are used to calculate different tumor sizes. The study group consists of twenty-two patients with different tumor types (meningioma, glioblastoma, abscess, metastases, and glioma). All patients were scanned and all the MRI data acquired at 1.5 Tesla with diagnosis histological confirmation. The results of this study show that the triangle mathematical method (method -3 ) is more accurate than the other compared manual methods. The correlation shows a linear relationship between the mean values of method-1 and method-2 has $R 2=$ 0.7975 and the $\mathbf{P}$-value $=\mathbf{0 . 0 2 6}$ indicates the significance difference between method-1 and method- 2 . Moreover, the correlation between method-1 and method-3 has R2 $=0.9367$ and the $P$-value $=0.003$ that indicates high significance between them. The comparative study of the three manual methods demonstrated that triangle manual method (method-3) has significant approach to be used in medical diagnosis. Additionally, method-3 can indicate which part of the tumor tissue is totally damaged depending on the image parameters. Therefore, the triangle manual method can be considered as an accurate step in the diagnostic process.
\end{abstract}

Keywords: MRI, Brain tumor, Heron’s formula, Image segmentation

\section{Introduction}

The conventional magnetic resonance imaging (MRI) techniques are based on the fundamental physical properties of tissue's protons, such as longitudinal relaxation time (T1), transverse relaxation time (T2), proton density (PD), and delayed permeability, which produce image contrasts [1]. Brain tumor segmentation aims to determine the tumor tissue's volume (even solid or active tumor with edema or necrosis) from normal brain tissues (gray matter, white matter, and cerebrospinal fluid). Many studies in the field of medical imaging and soft computing have provided extensive literature on brain tumor segmentation [2-5].
Clinical acceptance of segmentation techniques depends on the simplicity of the segmentation and the degree of user supervision. Computer-assisted surgical planning and advanced image-guided technology are increasingly used in neurosurgery [ 6]. Thus, tumor size is an important predictor of local tumor control in patients suffering from brain tumor cancer and treated with radio- or chemotherapy. Tumor growth curves were formulated, and then programmed for a digital computer. Afterward, they were subjected to widespread use and statistical evaluation [7]. A recent study suggested that MRI is a widely-used technique, especially in brain imaging, due to its

Corresponding Author: elywa2006@yahoo.com

DOI: 10.21608 /ajnsa.2018.6510

(C)Scientific Information, Documentation and Publishing Office (SIDPO)-EAEA 
soft tissue contrast, noninvasiveness, and highquality medical imaging. An important use of MRI data is tracking the size of a brain tumor as it responds to treatment $[8,9]$.

The availability of three-dimensional (3D) anatomical accuracy methods substantially improves spatial information concerning the relationships of critical structures (e.g., functionally significant cortical areas, vascular structures) and disease $[10,11]$. The pre- and postoperative tumor volumes are often based on the surgeon's impression or by measuring the greatest axis of the tumor in $\mathrm{x}-, \mathrm{y}$ - and z-direction [12].

Early detection of a minor tumor size change is difficult using traditional visual and clinical examination measurements. Although timeconsuming, the general medical manual methods are the standard approach for quantitative measurement of human tumor and are used in several hospitals $[13,14]$.

Currently, however, there is no widely accepted method in clinical practice for the assessment of tumor volume from MR images [15]. In a former study, an approximation of tumor area in the single MR slice with the largest contiguous was used. Herein, we quantitatively compared two manual methods and an automatic method to measure tumor volume from MR image data acquired at 1.5 Tesla (T) clinical platforms.

\section{MATERIALS AND METHODS}

\section{Patient population}

The study group consists of 22 patients with different tumors without control, detected by 150 images obtained from $1.5 \mathrm{~T}$ scanners. All the patients had histologically confirmed the diagnosis. The patients in this study were of different age groups (mean age: $21.5 \pm 10.03$ years). The patients were informed about the duration of the examination, their position, and the importance of being motionless. Prior to the study, informed consent was obtained from all the patients according to procedures approved by the institutional review board.

\section{MRI acquisition}

All the measurements were performed on a $1.5 \mathrm{~T}$ whole-body MRI system (Philips Medical Systems). In this study, an 8-channel coil was used for in vivo measurements. The vital difference between the surface and the volume coils is that the volume coils are always much larger than surface ones and can completely surround human head. Although the surface coils have higher signal-to-noise ratio because they only detect noise from the region of interest. They were usually used as receive coils but they make poor transmission coils because they have poor RF homogeneity, even over their region of interest._On the other hand, volume coils are used both as receiver and transmitter and can acquire signals from deep brain regions.

MR images were obtained with the patients in the supine position using the standard head coil. The examination was carried out before contrast administration. A scout sagittal T1-weighted view was obtained to verify the precise position of the patient and to act as a localizer for subsequent slices. Thereafter, multiple pulse sequences were used to acquire axial images followed by coronal and/or sagittal images based on the location of the pathology encountered. In midline lesions, sagittal planes were used while in laterally located lesions coronal images were more helpful.

The contrast medium used was either Omniscan or Magnevist (Gadolinium-Diethylene Triamine Penta acidic acid, Gd-DTPA), which was administrated intravenously at a dose of $0.1 \mathrm{mmol} / \mathrm{kg}$ body weight. T1-weighted image (T1-WI) was obtained immediately after the end of contrast injection. All the cases were examined using the following protocol: $\mathrm{FOV}=18-24 \mathrm{~cm}$ in axial images and 22-25 cm in coronal images; matrix size (frequency $\times$ phase) $265 \times 204$; and slice thickness $=5-6 \mathrm{~mm}$ with $2 \mathrm{~mm}$ interval for all the sequences. Young children were sedated using Ketalar (ketamine hydrochloride; $1 \mathrm{mg} / \mathrm{Kg}$ intravenously [IV] or 5-7 mg/Kg intramuscularly [IM]) to avoid motion artifacts.

\section{Tumor volume measurement}

The images obtained from all the patients were rated in one batch for three mathematical measurements. In this work, five non-radiologists performed the tumor volume measurements to compare inter- and intra-operator reliability. We needed at least two individuals who were available to perform the repetitive measurements. For all the methods, only the enhancing mass of the tumor 
was included in the volume calculation. The nonenhancing parts within the tumor (i.e., areas of tumor necrosis) were also traced, and their volumes were excluded from the total tumor volume.

Tumor volumes were determined manually by outlining the areas of the tumor multiplied by the image slice thickness and the interval gap in each slice using a MATLAB code. In the current study, the mathematical methods for tumor volume measurement were:

Method 1:

$$
A=(\text { height } \times \text { width })
$$

Method 2:

For a non-self-intersecting polygon with $\mathrm{n}$ vertices $x_{i}, y_{i}(i=1$ to $\mathrm{n})$, the area is given by

$$
A=\frac{1}{2}\left|\sum_{i=0}^{n-1} x_{i} y_{i+1}-x_{i+1} y_{i}\right|
$$

Method 3:

Our mathematical method that divides the region of interest into several triangles then measure the total volume, $A_{t}=\left(\sum_{j=1}^{m-1} A_{i}\right), \mathrm{j}=1: \mathrm{m}, \mathrm{m}$ is the number of triangles and its area is

$$
A=s \sqrt{(s(s-a))(s-b)(s-c)}
$$

Where $s=(a+b+c) / 2$ and $a, b, c$ the lengths of triangle according to Heron's Formula [16].

The slice tumor volume was estimated using (Volume $=$ area $\times$ slice thickness) for each slice, and then the summation of all volumes in the three directions was evaluated. The standard deviation SD has calculated for each case, and then the standard error of mean determined.

\section{Results and Discussion}

Human tumor shape was assumed to be a hemiellipsoid using method-1 in glioma [17], breast [18], prostate [19, 20], as well as the available segmentation methods.

Fig. (1) shows the three mathematical evaluation methods applied on acquired magnetic resonance enhanced images of the same patient. It also illustrates the accuracy of using different manual measuring methods to detect tumor size. Fig. (1A) shows method- 1 by which the tumor's area of each slice calculated by height times width. Fig. (1B) shows the polygon surrounding the tumor for each slice (mezhod-2). Fig. (1C) shows our triangle method (method-3) where the tumor divided into a number of triangles, in which each triangle should have an area $\left(A_{1}\right)$ and intensity $\left(I_{1}\right)$; hence, the one triangle intensity calculated using equation 4 .

$$
I_{1}=\frac{I_{t} A_{1}}{A_{t}}
$$

Radiologists use the manual method 1 as shown in Fig. (1 A) because it does not consume much time for analysis. However, it has a lower accuracy compared to semiautomatic or automatic methods. Even though the new software programs can perform the volume calculation automatically [25], the manual methods is still used in several medical centers worldwide because the users prefer to determine the parts of tumor manually. Therefore, method 3, that depends upon the division the tumor into a few number of the triangle, is used to define which part of cells is fully damaged and which is partially damaged depending on the intensity of each part. In Fig. (1), the total area of tumor was divided into eleven triangular areas; area number 3 has the highest relative intensity (relative intensity $=$ intensity of each triangle area/total intensity of total tumor area) than the other areas. This means that the cells in this region were more damaged than the cells in other areas. This results may be useful in the diagnostic process and the in the surgery.

Table (1) shows the P-values and type of the examined tumor. The tumor volume measurements of 22 patients with different cases (meningioma, glioblastoma, abscess, metastases, and glioma) have been done by using the one-way ANOVA test for each case.

The following classification is based on the medical image information, which is used to achieve the segmentation; pixel-based method, region-based methods, edge-based methods, based methods [21 - 24]. Columns 3, 4 and 5 indicate the mean \pm SD using method 1 , method 2 and method 3 , respectively. The three applied mathematical methods were compared in terms of brain tumor types (glioblastoma, meningioma, metastases, abscess, and glioma).

Fig. (2 A) shows the mean values of tumor sizes of eight patients with Meningioma tumor of different 
volumes. The three methods were applied on the same patient's data. The mean \pm SD values of method- 1 are $12.28 \pm 2.73$, for method-2 are 7.21 \pm 2.45 , and for method-3 $7.07 \pm 2.27$ respectively. The change percentage between method- 1 and method-2 (reference method) is increased by $12 \%$ and between method-3 and method-2 (reference method) is decreased by $6 \%$. This means that there is no more normal tissue included in the tumor boundary and hence the radio dose will be more affected in radiotherapy. The three applied mathematical methods were compared in terms of brain tumor types (glioblastoma, meningioma, metastases, abscess, and glioma) as shown in Fig. (2A, B, C, D, E), respectively. Fig. (2B) shows determination of meningioma brain tumors of eight patients. Method-2 and method-3 have significant difference with respect to method- $1(\mathrm{p}<0.05)$, the mean \pm SD values are $8.95 \pm 2.17,6.69 \pm 1.22$ and $6.56 \pm 0.90$ for method-1, method-2 and method-3 respectively. (2C) Indicates mean volume of metastases brain tumors of three patients. Method2 and method-3 have significant difference with respect to method- $1(p<0.05)$, where the mean \pm SD values are $12.45 \pm 3.19,8.15 \pm 2.76$ and $5.88 \pm$ 1.69for method-1, method-2 and method-3 respectively. (2D) Shows determination of three glioma patients. Method-2 and method-3 have significant difference respect to method-1(p < $0.05)$, the mean $\pm \mathrm{SD}$ values are $8.76 \pm 2.11,7.59 \pm$ 1.33 and $5.36 \pm 1.16$ for method-1, method-2 and method-3 respectively. (E) Shows determination of abscess tumors of four patients. Method-2 and method-3 have significant difference respect to method-1( $\mathrm{p}<0.05)$, the mean \pm SD values are $8.99 \pm 2.76,6.23 \pm 2.03$ and $5.46 \pm 1.04$ for method1 , method-2 and method-3 respectively.

Table (2): shows the columns for the sums of squares (SS), degrees of freedom (DF), Mean squares (SS/df), F-Statistic, and p-value. Moreover, it indicates that there was a significant variation between methods i.e., triangle manual method (method 3) is useful for tumor size measurement at the $\mathrm{p}<0.05$ level for the three conductions $[F(2,63)=3.933, p=0.001]$ as shown in Table (2).

Table (3) shows that the mean values of the three methods are different. There are slightly significant differences between methods 1 and 2 and accept significance between methods 1 and 3 , but the difference in means between methods 2 and 3 are closely the same.

Since the significance means that at least 95\% chance of being is achieved, another posttest (posttest t-test) is required to indicate the significant difference between methods. Therefore we can use a new significance point that is 0.05 divided by a number of comparisons, in our work, there are only three methods, and thus the new significance point is 0.0167 . Here, the significance occurs if and if only the p- value is less than 0.0167. A comparison between method-1 and (method-2, method-3) is illustrated in Table (3) which indicates that there is a significant difference between method 1 and method 3; on the other hand, there is no significant difference between method-1 and method-2.

Fig. (3A) shows the linear relationship between the mean values of methods 1 and 2 . Additionally, the correlation between methods 1 and 3 is presented in Fig. (3B). The correlation shows a linear relationship between the mean values of methods 1 and 2 with R2 $=0.7975$ and the P-value $=0.062$ that indicates that there is no significant difference between the two methods. Moreover, the correlation between methods 1 and 3 with R2 = 0.9367 and the $\mathrm{P}$-value $=0.003$ indicates a strong linear relationship in method 1 , which is considered the reference method. Thus, the triangle mathematical method 3 is highly accurate than the other compared manual methods especially Method 1 for determining the tumor size. The linear correlation between methods- 1 and method3 represents a positive relationship that indicates the significance of using method 3 to detect the volume and shape of different types of human brain tumors. Method-3 enables radiologists to evaluate the human brain tumor manually with higher accuracy than the traditional methods (method-1). Additionally, the volumetric measurement including the conventional manual method, semi-automatic method (using Philips software), and the triangle manual method (using Heron's formula), can be routinely applied in MRI systems. Moreover, the comparative study of the three methods shows that in about $80 \%$ of the studied cases, the triangle manual method had the lowest standard error of mean (SEM) than the other two methods; accordingly, it can be useful for radiologists. 


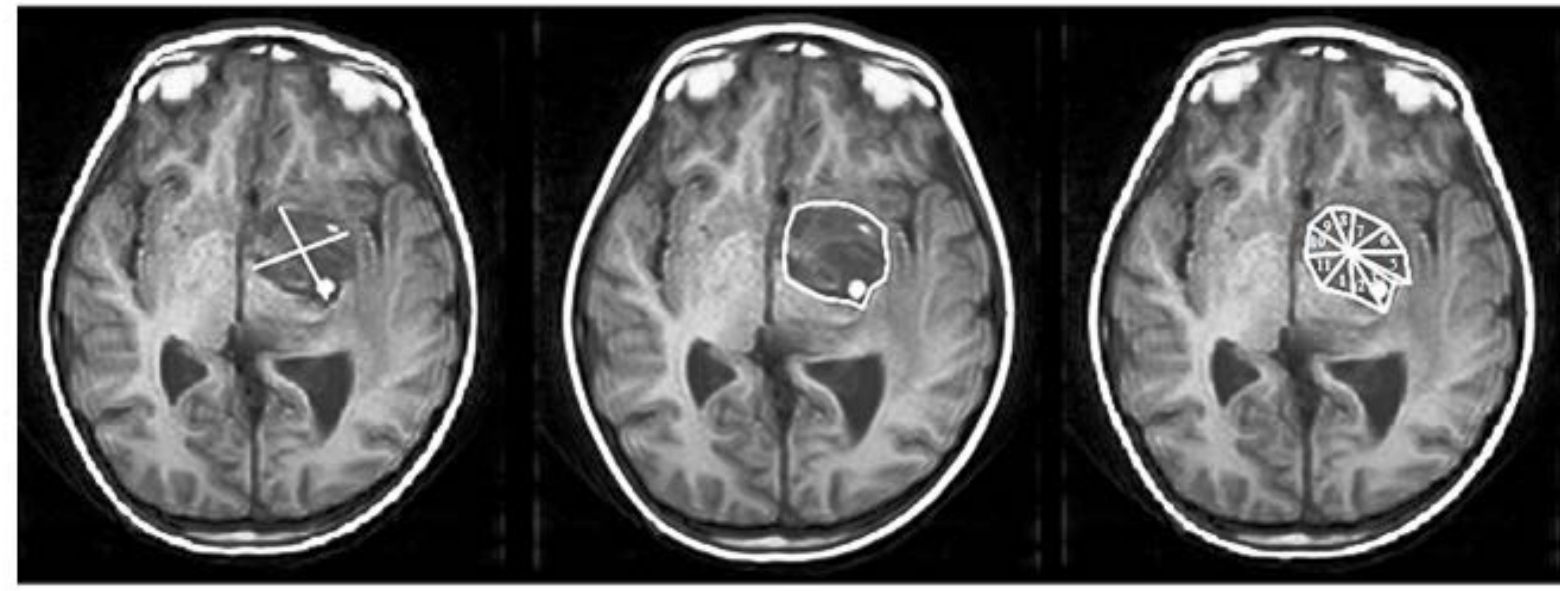

(A)

(B)

(C)

Fig. (1): Axial T1-enhanced MR image; (A) the area of tumor in two dimensions using method 1, in which the tumor area is determined by two lines appearing in white color; (B) a polygon area estimated by method 2; our method (method 3) as shown in (C), shows many triangles fill the tumor region.

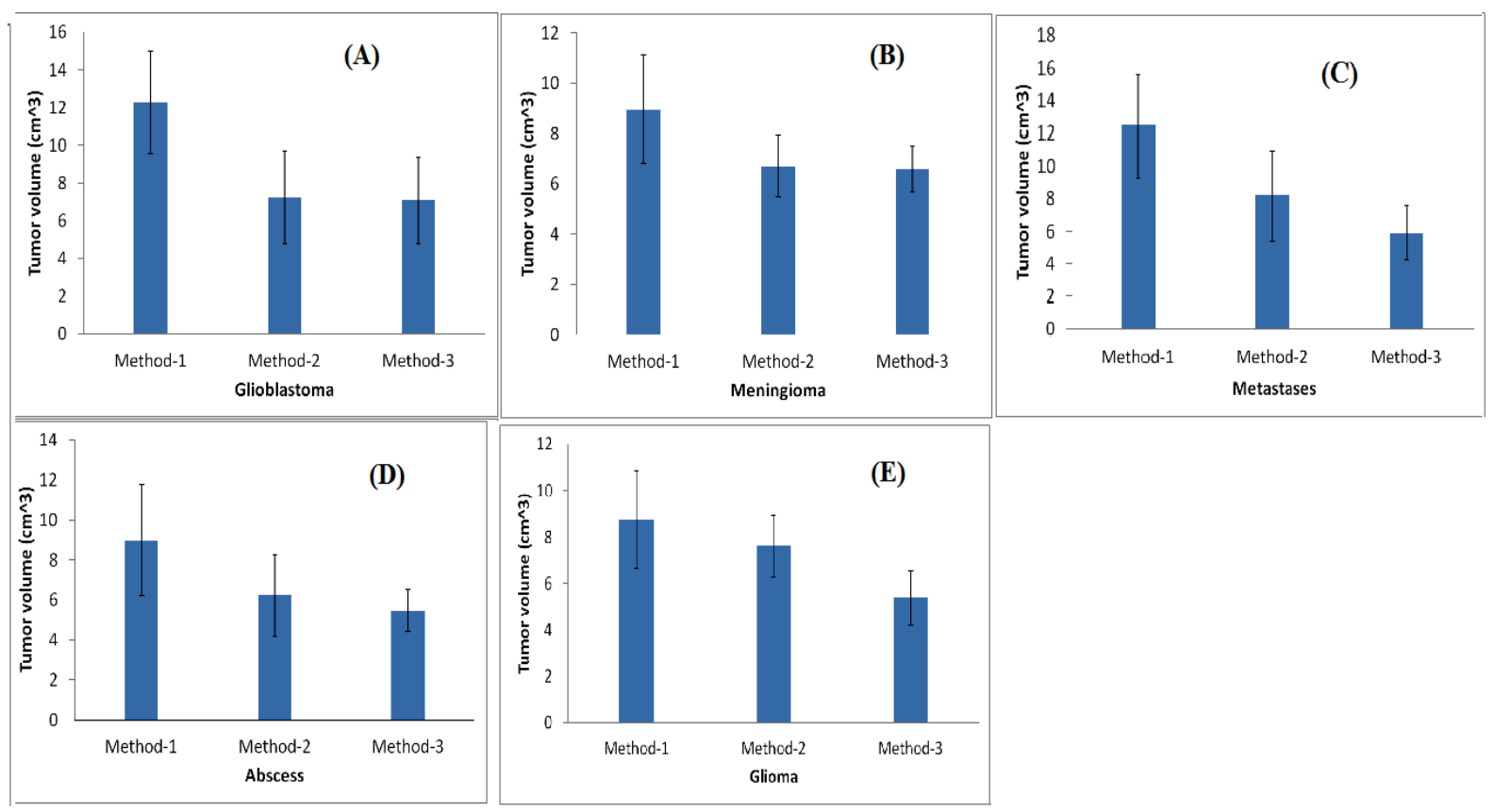

Fig. (2): the mean \pm SD of applied mathematical methods comparison, (A) four Glioblastoma patients using (B) eight Meningioma patients (C) three Metastases patients (D) four Abscess patients (E) three Glioma patients

Table (2): one way ANOVA test indicates p-value between methods that of the mean of 22 patients

\begin{tabular}{lrrrrr}
\hline \multicolumn{1}{c}{ Source of Variation } & SS & $d f$ & \multicolumn{1}{c}{ MS } & F & P-value \\
\hline Between Groups & 144.574 & 2 & 72.287 & 3.933 & 0.001 \\
Within Groups & 1157.842 & 63 & 18.378 & & \\
& & & & & \\
Total & 1302.417 & 65 & & & \\
\hline
\end{tabular}


Table (3): Comparison between methods both each other, source of variation, p-value from one-way ANOVA test and posttest t-test

\begin{tabular}{lccc}
\hline Source of Variation & P-value & new significance point & significance \\
\hline Between three methods & 0.001 & $\mathrm{p}<0.0167$ & yes \\
Between method 1 and method 2 & 0.062 & $\mathrm{p}>0.0167$ & no \\
Between method 1 and method 3 & 0.003 & $\mathrm{p}<0.0167$ & yes \\
\hline
\end{tabular}
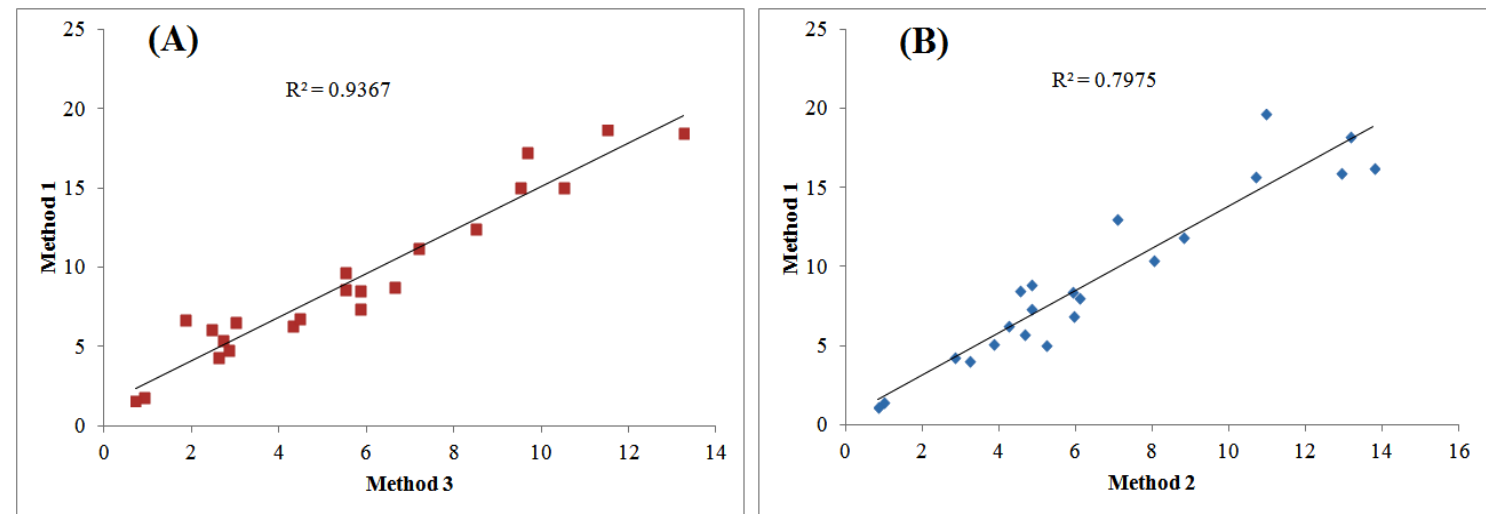

Fig. (3): The linear correlation of standard error of mean between the three mathematical methods. (A) the linear relationship between SEM of methods 1 and 2. (B) The linear relationship between SEM of methods 1 and 3

\section{Conclusion}

Currently, there is no widely accepted method in clinical practice for the measurement of tumor volumes using MRI. Approximation of tumor area in the single MR slice with the largest contiguous was discussed in this study. Our results showed that the volumetric measurements using methods 1,2 and 3 can be routinely applied in MRI systems, but the accuracy of tumor measurement was higher in method-3 compared to method-1. Moreover, the comparative study of the three methods revealed that method 3 had the lowest SEM. Therefore, our method can be used to calculate any human brain tumor with different sizes, shapes, and types, and it might be beneficial for radiologists.

Acknowledgements: I would like to thank all the staff member of the MRI unit at the Zagazig University Hospital.

\section{References}

1- Young GS. Advanced MRI of adult brain tumors. Neurologic clinics 2007;25(4):947-73.

2- Cartes-Zumelzu F.,Stavrou I, Castillo M, Eisenhuber E, Knosp E, Thurnher MM. Diffusionweighted imaging in the assessment of brain abscesses therapy. American journal of neuroradiology 2004; 25(8):1310-7.

3- Lai PH, Ho JT, Chen WL, Hsu SS, Wang JS, Pan HB, et al. Brain abscess and necrotic brain tumor: discrimination with proton MR spectroscopy and diffusion-weighted imaging. American Journal of Neuroradiology 2002; 23(8):1369-77.

4- Buckner JC, Brown PD, O'Neill BP, Meyer FB, Wetmore CJ, Uhm JH. Central nervous system tumors. InMayo Clinic Proceedings 2007; 1271-86.

5- Sachdeva J, Kumar V, Gupta I, Khandelwal N, Ahuja CK. A novel content-based active contour model for brain tumor segmentation. Magnetic resonance imaging 2012;30(5):694-715.

6- Moriarty TM, Kikinis R, Jolesz FA, Black PM, Alexander E. Magnetic resonance imaging therapy Intraoperative MR imaging. Neurosurgery Clinics of North America 1996;7(2):323-31.

7- Dethlefsen LA, Prewitt JM, Mendelsohn ML. Analysis of tumor growth curves. Journal of the National Cancer Institute 1968;40(2):389-405. 
8- Hao X, Bruce C, Pislaru C, Greenleaf JF. A novel region growing method for segmenting ultrasound images. InUltrasonics Symposium IEEE 2000 ;2: 1717-1720.

9- Averette HE, Ford Jr JH, Dudan RC, Girtanner RE, Hoskins WJ, Lutz MH. Staging of cervical cancer. Clinical obstetrics and gynecology 1975;18(3):21532.

10- Nakajima S, Atsumi H, Bhalerao AH, Jolesz FA, Kikinis R, Yoshimine T, Moriarty TM, and et al., Computer-assisted surgical planning for cerebrovascular neurosurgery. Neurosurgery 1997;41(2):403-10.

11- Hu X, Tan K, Levin D, et al.Threedimensional magnetic resonance images:application to neurosurgical. J Neurosur. 1990:72; 433-40.

12- Kikinis R, Gleason PL, Moriarty TM, Moore MR, Alexander III E, Stieg PE, et al. Computer-assisted interactive three-dimensional planning for neurosurgical procedures. Neurosurgery 1996;38(4):640-51.

13- Clarke LP, Velthuizen RP, Clark M, Gaviria J, Hall $\mathrm{L}$, Goldgof D, et al. MRI measurement of brain tumor response: comparison of visual metric and automatic segmentation. Magnetic resonance imaging 1998;16(3):271-9.

14- Joe BN, Fukui MB, Meltzer CC, Huang QS, Day RS, Greer PJ, et al. Brain Tumor Volume Measurement: Comparison of Manual and Semiautomated Methods 1. Radiology 1999;212(3):811-6.

15- Durrance, FY., Fletcher GH, Rutledge FN. Analysis of central recurrent disease in stages I and II squamous cell carcinomas of the cervix on the intact uterus. American Journal of Roentgenology 1969;106(4):831-8.

16- Raifaizen, Claude H. "A Simpler Proof of Heron's Formula". Mathematics Magazine 1971; 44 (1): $27-$ 28.

17- Schmidt KF, Ziu M, Schmidt NO, Vaghasia P, Cargioli TG, Doshi S, et al. Volume reconstruction techniques improve the correlation between histological and in vivo tumor volume measurements in mouse models of human gliomas. Journal of neuro-oncology 2004;68(3):207-15.

18- Wapnir IL, Barnard N, Wartenberg D, Greco RS. The inverse relationship between microvessel counts and tumor volume in breast cancer. The breast journal 2001;7(3):184-8.

19- Sosna J, Rofsky NM, Gaston SM, DeWolf WC, Lenkinski RE. Determinations of prostate volume at 3-tesla using an external phased array coil: comparison to pathologic specimens1. Academic radiology 2003;10(8):846-53.

20- Egorov V, Ayrapetyan S, Sarvazyan AP. Prostate mechanical imaging: 3-D image composition and feature calculations. IEEE transactions on medical imaging 2006;25(10):1329-40.

21- Mayr NA, Taoka T, Yuh WT, Denning LM, Zhen WK, Paulino AC, et al. Mannel RS. Method and timing of tumor volume measurement for outcome prediction in cervical cancer using magnetic resonance imaging. International Journal of Radiation Oncology Biology Physics 2002;52(1):14-22.

22- Clarke LP, Velthuizen RP, Camacho MA, Heine JJ, Vaidyanathan M, Hall LO, Thatcher RW, Silbiger ML. MRI segmentation: methods and applications. Magnetic resonance imaging 1995;13(3):343-68.

23- Heimann T, Meinzer HP. Statistical shape models for 3D medical image segmentation: a review. Medical image analysis 2009;13(4):543-63.

24- Sosna J, Rofsky NM, Gaston SM, DeWolf WC, Lenkinski RE. Determinations of prostate volume at 3-tesla using an external phased array coil: comparison to pathologic specimens1. Academic radiology 2003;10(8):846-53.

25- Meier R, Knecht U, Loosli T, Bauer S, Slotboom J, Wiest R, et al. Clinical Evaluation of a Fullyautomatic Segmentation Method for Longitudinal Brain Tumor Volumetry. Scientific reports 2016. 